\title{
Promoting community reintegration using narratives and skills building for young adults with stroke: a protocol for a randomised controlled trial
}

\author{
Suzanne Hoi Shan Lo ${ }^{1 *}$, Janita Pak Chun Chau ${ }^{1}$, Kai Chow Choi ${ }^{1}$, Edward Wai Ching Shum², \\ Jonas Hon Ming Yeung ${ }^{3}$ and Siu Hung Li ${ }^{4}$
}

\begin{abstract}
Background: Stroke in adults aged between 18 and 64 years old is increasing significantly worldwide. Studies have reported that this group of young stroke survivors encounters enormous difficulties reintegrating into their social roles. Individualised discussions with healthcare professionals and learning from other survivors are imperative for them to reconstruct their identities after stroke. There is also great demand for community support during their chronic stage of recovery to help them rebuild life skills to promote reintegration.

Methods/design: This is a randomised controlled trial to investigate the effects of a 24-week Narrative and Skillsbuilding Intervention (NSI) on young stroke survivors' community reintegration and psychosocial outcomes. A total of 208 adults aged 18-64 years old with a first-ever or recurrent ischaemic or haemorrhagic stroke and have been discharged home will be recruited and randomly assigned to receive usual care or usual care with NSI. The NSI is grounded in Narrative Theory and Bandura's principles of Self-efficacy and Outcome Expectation, consisting of successive eight individual sessions over six months delivered by a trained facilitator (a registered nurse).

Participants will be facilitated to narrate their survival experiences and rebuild core life skills. Videos of peer young stroke survivors' experiences of recovery will be provided. Outcomes including community reintegration, depressive symptoms, health-related quality of life, self-efficacy, outcome expectation and satisfaction with performance of selfmanagement behaviours will be measured before (T0) and immediately after NSI (T1), then six (T2) and 12 months after NSI (T3). Generalised estimating equations models will be used to compare the differential changes in outcomes across time between the two groups. Focus group interviews will be conducted with the facilitator at T1 and with the participants in the intervention group at $\mathrm{T} 1$ and $\mathrm{T} 3$.

Discussion: This study will evaluate the short and long-term effects of a theory-based NSI on young stroke survivors' community reintegration and establish a new model of community reintegration after stroke to inform future research. The results will also provide valuable evidence to develop clinical guidelines for young stroke survivors' community reintegration.

(Continued on next page)
\end{abstract}

\footnotetext{
* Correspondence: suzannelo@cuhk.edu.hk

${ }^{1}$ The Nethersole School of Nursing, Faculty of Medicine, Chung Chi College,

The Chinese University of Hong Kong, Shatin, N.T., Hong Kong SAR, China

Full list of author information is available at the end of the article
}

\section{$\triangle B M C$}

(c) The Author(s). 2021 Open Access This article is licensed under a Creative Commons Attribution 4.0 International License, which permits use, sharing, adaptation, distribution and reproduction in any medium or format, as long as you give appropriate credit to the original author(s) and the source, provide a link to the Creative Commons licence, and indicate if changes were made. The images or other third party material in this article are included in the article's Creative Commons licence, unless indicated otherwise in a credit line to the material. If material is not included in the article's Creative Commons licence and your intended use is not permitted by statutory regulation or exceeds the permitted use, you will need to obtain permission directly from the copyright holder. To view a copy of this licence, visit http://creativecommons.org/licenses/by/4.0/ The Creative Commons Public Domain Dedication waiver (http://creativecommons.org/publicdomain/zero/1.0/) applies to the data made available in this article, unless otherwise stated in a credit line to the data. 
(Continued from previous page)

Trial registration: ClinicalTrials.gov identifier: NCT04560140, registered on 23 September, 2020.

Keywords: Stroke, Narration, Social participation, Self-management, Randomised controlled trial

\section{Background}

The global prevalence of stroke in adults aged 20-64 years old doubled from 1990 to 2013 [1]. International guidelines recommend promoting community reintegration as a rehabilitation priority for stroke survivors [2, 3]. Community reintegration refers to one's ability to resume or adapt one's roles and responsibilities and to actively participate in social activities after a disabling illness [4]. Studies have reported that this group of young adults with stroke encounters substantially greater difficulties reintegrating into their social roles compared to older adults. Common challenges include maintaining family relationship and a social network, resuming leisure activities, or returning to work $[5,6]$. Ageappropriate interventions to facilitate young stroke survivors' acceptance of their functional limitation and development of life skills to reconstruct their roles for better community reintegration are warranted.

Previous studies have consistently reported the complex and chronic nature of young stroke survivors' community reintegration [5-8]. A review of the literature on survivors aged 18-65 years old found that the abrupt onset of stroke was often stigmatised as a disease of old age, and that their ability to perform self-care activities was consequentially compromised, which significantly disrupted their sense of self, role and relationship. They had a loss of control and an increased sense of uncertainty in their life after stroke [5]. A qualitative study of 17 community-dwelling survivors aged $23-55$ years old reported that they might develop an adapted sense of self several years after the stroke event. However, it has been suggested that these survivors need guidance and support to reflect on their experiences, re-appraise their strengths and re-adjust their personal values to reconstruct their identities and roles [6]. A qualitative study of 22 stroke survivors who were aged 20-61 years old and about six months to nine years after stroke found that their struggles of inability to fulfil their role expectations or maintain intimate relationships resulted in emotional shortcomings such as feelings of isolation or helplessness. The results highlighted the need for providing survivors with opportunities to narrate their experiences outside their homes and offering support in the later phase of recovery to prevent psychosocial problems [7]. Another study of 12 women aged 18-50 years old has also highlighted the young stroke survivors' unmet needs, including a lack of access to age-appropriate information, inadequate professional support regarding life skills training, limited dialogue with healthcare professionals and underuse of peer learning to support the continual reintegration process [8].

Personal or illness experience narratives have been examined in previous studies to help stroke survivors reestablish their identity and roles through meaningmaking, bridging between the present situation and the ideal future life, and exploring opportunities for change [9-11]. A feasibility study of 17 survivors aged 33-89 years old reported that those receiving a nurse-led intervention using narratives appreciated the opportunities to share and re-create their stroke experience with a healthcare professional. Following Narrative Theory, the participants were supported to reflect on their experience through this act of narrating. The narrative facilitated their reflection and self-understanding. It stimulated them to envision for future changes and make adjustment in their lives after stroke [10]. A onegroup study of 27 survivors aged 44-73 years old with post-stroke aphasia found that a biographic-narrative intervention was associated with potential benefits in health-related quality of life (HRQoL) and mood at three months post-intervention. Participants were asked to share their daily life experiences after stroke to reshape their identity. They were also facilitated to learn from peers and increase social contact via discussing health or leisure time issues during group sessions [11].

Skills-building is vital to improve stroke survivors' ability to plan, solve problems and set personal goals for better community reintegration [12]. Three experimental studies examined the effects of self-management interventions aimed at promoting participation in daily life after stroke. The interventions, led by an occupational therapist, focused on home modifications and developing survivors' self-management skills, including problem solving, decision making, action planning, resource utilisation and partnership with care providers [13-15]. Among the studies, one randomised controlled trial (RCT) of 185 adults with mild to moderate stroke found significant improvements in health-related and participation self-efficacy in daily life activities immediately after completing the 12-session group and community-based intervention [13]. A follow-up quasi-experimental study of 17 survivors found significant improvement in community reintegration upon completion of a 6-session intervention enriched with components for problem solving and goal setting [14]. A randomised pilot study of 15 survivors also reported a clinically meaningful 
change in social participation at six months after receiving a participation-focused individual-based programme that included one pre-discharge and five post-discharge home visits [15]. Several systematic reviews have suggested that problem-solving skills are likely to facilitate self-management after stroke [12, 16, 17]. Contrastingly, an RCT of 62 stroke survivors reported significant improvements in depressive symptoms and anxiety among those who received a solution-focused therapy compared with the control group [18]. Two qualitative studies of 12 stroke survivors also suggested doing everyday activities at home developed skills in managing role changes through mastery experiences and negotiation with spouses $[19,20]$.

There have been limited studies that examined interventions for promoting community reintegration after stroke, and young stroke survivors are often under-represented in these studies. Moreover, grounding the design, development and evaluation of such a complex intervention in a theoretical framework is important to explain the mechanisms underlying the changes in outcomes [21]. More investigations on the best theoretical frameworks that consider a person's interpretation of experiences and self-efficacy relating to behavioural change are needed. There are an increasing number of studies that have used narratives as an approach to help stroke survivors to understand their experience of stroke and have showed positive results for psychological well-being. More evidence on the effects of using narratives in promoting the community reintegration of young stroke survivors would be worthwhile. Young stroke survivors need to develop life skills to address their internal struggles and the challenges they encounter during the return to their family and social roles. There has been no consensus, however, on the core skills for promoting community reintegration in young stroke survivors.

This study aims to investigate the effects of a 24-week Narrative and Skills-building Intervention (NSI) on community reintegration, self-efficacy, outcome expectation, satisfaction with performance of self-management behaviours and psychosocial outcomes among young stroke survivors. We hypothesise that the participants in the intervention group, compared with the control group, will demonstrate all of the following at immediately, 6 months and 12 months post-intervention: a) Significant increase in community reintegration with respect to baseline; b) Significant improvements in depressive symptoms, HRQoL and satisfaction with performance of self-management behaviours with respect to baseline, and c) Significant increases in self-efficacy and outcome expectation of performing self-management behaviours with respect to baseline.

\section{Methods/design Study design}

A multi-centred, assessor-blinded, two-arm RCT will be conducted. The outcomes will be measured at baseline (T0), immediately post-intervention (T1), and 6 months (T2) and 12 months post-intervention (T3) (Fig. 1).

\section{Setting}

Participants will be recruited from three acute public hospitals, and community-based organisations serving people with chronic conditions including stroke in Hong Kong. The three hospitals follow the same stroke care pathway endorsed by the Hong Kong Hospital Authority. All sessions of the NSI and all of the assessments will be conducted in the participant's homes or a community centre.

\section{Participants}

Individuals will be recruited if they: (1) are 18-64 years of age, (2) have a clinical diagnosis of first-ever or recurrent ischaemic or haemorrhagic stroke, (3) are living at home after discharge from hospital, (4) have a Montreal Cognitive Assessment (MoCA) score $>2$ nd percentile, (5) have a modified Rankin Scale (mRS) score $\geq 3$ (moderate disability), (6) can communicate in Cantonese, and (7) are able to attend all NSI sessions.

Individuals will be excluded if they 1) have been diagnosed with transient ischaemic attack, subdural or epidural haemorrhage [22], 2) have experienced cerebrovascular events due to tumours or head trauma, 3) have been diagnosed with a mental condition such as depression, schizophrenia, bipolar or personality disorder, 4) demonstrate incomprehensible speech or difficulty in comprehending conversations, or 5) have received a selfmanagement programme in the past.

\section{Sample size estimation}

The sample size is estimated based on the outcomes of community reintegration, self-efficacy and outcome expectation. In our previous interventional study [23], the effect sizes of a stroke self-management programme on community reintegration, self-efficacy and outcome expectation of performing self-management behaviours were $0.44,0.55$ and 0.53 , respectively. Using the power analysis software PASS 16 (NCSS, LLC. Kaysville, Utah, USA), a sample size of 83 participants per study arm is estimated to give this two-arm RCT $80 \%$ power at a two-sided 5\% level of significance to detect an effect size as small as 0.44 on the primary outcome between the control and intervention groups at a post-intervention time point. Further allowing for a potential attrition rate of up to 20\%, 208 participants, with 104 per group, will be recruited. 


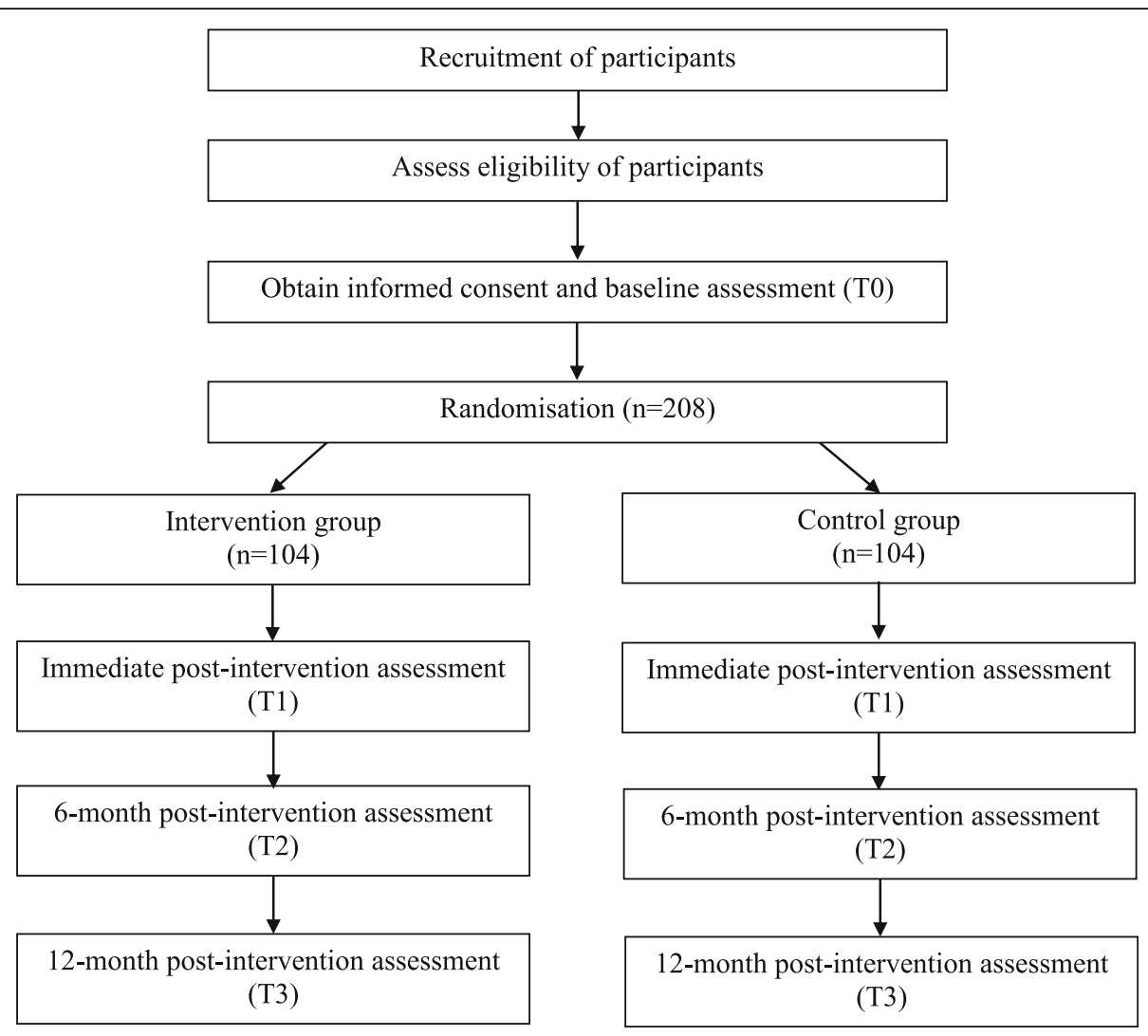

Fig. 1 Study flow diagram

\section{Randomisation and blinding}

The participants will be randomly allocated to either the intervention (I) or the control (C) group at 1:1 ratio after they have provided consent and baseline data. Block randomisation in blocks of ten, except the last block, will be used. An independent statistician will generate a random sequence of grouping identifiers (I or C) using a random list generator (www.randomization.com). According to the sequence, a grouping identifier will be placed into an opaque, identical, sealed and sequentially numbered envelope for concealed allocation. All participants will be pooled into the same enrolment list regardless of their recruitment venues. A research assistant will open the envelopes sequentially according to the participants' time of enrolment and record the grouping. Another research assistant who will conduct the baseline and follow-up assessments and data entry will have no knowledge of the participants' group assignments. Blinding is not possible for the participants and the facilitator who will deliver the NSI, due to the nature of the intervention. The research assistant, who will conduct qualitative evaluation with the facilitator and participants' feedback on the NSI, will know the group allocations.

\section{Intervention}

The NSI aims to promote community reintegration of young stroke survivors and is designed on the basis of Narrative Theory [24] and Bandura's principles of Selfefficacy and Outcome Expectation [25]. The NSI will consist of eight face-to-face sessions (1.5-2 $\mathrm{h}$ each) to be delivered over 24 weeks by a registered nurse (facilitator). The NSI will blend narratives and skills-building in each session to support participants' reintegration into their post-stroke social roles. The narrative part will elicit the participants' narratives of their experiences of engaging in everyday social activities including social, leisure and work activities, and will be used to identify the participants' strengths, co-construct meanings of their experiences, re-structure self-identity and social roles, envision the future and explore opportunities for change. The skills-building part will develop the participants' abilities to apply the life skills learned in order to maintain health and sustain their engagement in social activities. Three types of life skills will be included: selfmanagement (goal setting, action planning, decision making, communication and utilisation of resources), outcome-expectation enhancing (reflection, solution focused) and self-efficacy enhancing (mastery, modelling, 
persuasion and reinterpretation of physical and emotional arousal) [12-20, 23].

Each session will have an assigned themed topic to articulate the narratives and skills-building. Each session is designed with a theme-related activity to arouse the participants' interest in narrating their experiences and discussing the assigned themed topic. Strategies informed by the theoretical framework and tailored to young stroke survivors' health needs will be adopted. The first three sessions will be foundational and conducted at the participants' home bi-weekly to engage the participants in applying the skills learned to perform everyday household and social activities. The participants' family members or caregivers will be invited to join these sessions. The remaining five sessions will be reintegrationenhancement sessions conducted in a private room at a community centre once every three weeks. These sessions will encourage the participants to address their struggles while reconstructing their self-identities and social roles. Each participant will be provided with a workbook with age-appropriate quick references for their narration and skills-building. Each participant will be given an online account to record their goals, action plans and daily accomplishments, and to retrieve 15 videos of other young stroke survivors sharing their experiences of reintegrating into social roles.

An expert panel consisting of two academics, one physician, one nurse consultant, two ward managers and one social worker will review the contents of the NSI. Their feedback will be incorporated in further revisions if deemed necessary. The revised NSI will be piloted with 10 potential young stroke survivors to assess their satisfaction and acceptability. Revisions will be made with consideration of the survivors' feedback.

\section{Usual care}

The usual care will include the usual stroke care services available to the participants during the study period. It will include, but not be limited to, medical consultations or rehabilitation services offered by the hospital or health organisations.

\section{Quantitative outcome measures \\ Primary outcome}

Community reintegration The Chinese version of the Reintegration to Normal Living Index (RNIL-C) will be used to assess the participants' level of community reintegration [26, 27]. It consists of 11 items in eight domains: mobility, self-care, daily work and school activity, recreational and social activities, family roles, personal relationships, presentation of self to others and general coping skills. Participants will be asked to rate the extent to which each item describes their situation on a scale from 1 - 'a small extent' to 5 - 'a great extent'. The total score is calculated by summation and normalised to give 100 with a total score range of 25 to 100 . A higher score indicates better community reintegration. The RNIL-C has high internal consistency (Cronbach's alpha $=0.92$ ) and good convergent validity [27].

\section{Secondary outcomes}

Depressive symptoms The Chinese version of the 15item Geriatric Depression Scale will be used to measure participants' depressive symptoms [28, 29]. Studies support its utility in younger adults ( $>18$ years old), with good diagnostic sensitivity and specificity [30, 31]. Each item represents symptoms of depression and describes a participant's condition in the preceding week; the participants answer each item with either 'yes' or 'no'. All items are summed (total score 0-15). A score of six or greater is a cutoff for depression. The scale has a Cronbach's alpha of 0.78 [29].

HRQoL Participants' HRQoL will be measured by the Chinese version of the Stroke-Specific Quality of Life Scale [32, 33], which has 47 items with 11 domains ranging from physical to psychosocial and participation. The items are about the health conditions of the participants and how much difficulty the participants have when doing everyday self-care tasks. The items are scored from 1 - 'strongly disagree/cannot do it' to 5 'strongly agree/no trouble'. Total score is yielded by summing all item scores (range 47-235): the higher the score, the higher the HRQoL. It has acceptable internal consistency (Cronbach's alpha: 0.63-0.93) and convergent validity [33].

Self-management behaviours The 11-item Chinese version of the Stroke Self-management Behaviours Performance Scale will be adopted to assess participants' satisfaction with the performance of self-management behaviours. Each item is scored using a range from 0 'very dissatisfied' to 10 - 'very satisfied'. Taking the sum of all item scores yields one total score (range 0-110), and the higher the score, the higher the satisfaction. This scale has a Cronbach's alpha of 0.93 [23].

Self-efficacy The Chinese version of the Stroke SelfEfficacy Questionnaire will be used to measure selfefficacy [34, 35]. It has 13 items, each is scored using a scale from 0 - 'no confidence' to 10 - 'very confident'. The items are about the participants' perceived extent of confidence in doing everyday activities and selfmanagement tasks. A total score is yielded by summing all items (range 0-130). A higher total score represents higher self-efficacy. The scale has acceptable internal 
consistency (Cronbach's alpha $=0.92)$ and convergent validity [35].

Outcome expectation The Chinese version of the Stroke Self-management Outcome Expectation Scale will be used to measure the participants' outcome expectation beliefs. It has 11 items, each rated using a scale from 0 - 'strongly disagree' to 10 - 'strongly agree'. The score of each item indicates the participants' confidence in the expected outcomes to occur. All item scores will be calculated by summation (total score $0-110$ ). A higher score represents higher outcome expectations. This scale has good internal consistency (Cronbach's alpha $=0.94)$ [23].

\section{Qualitative evaluation}

Focus group interviews with participants who received the NSI will be conducted at a community centre immediately and 12 months post-intervention. A convenience sample of 56 participants (7 per group) will be invited. The participants will be asked to share their experience and satisfaction with the NSI, views on usefulness of the NSI in initiating and supporting their engagement in valued everyday activities, family and social roles, met and unmet health needs, and areas for enhancement. The facilitator who delivered the NSI will also be interviewed using a semi-structured interview guide immediately after the completion of the intervention to elicit feedback on the facilitators of and barriers to implementation of the NSI.

\section{Demographic and clinical information}

Data will be recorded on the participants' age, gender, educational level, marital status, occupation, current financial aids received, type of housing, living condition, past and present medical history, and assistive aids used. Scales such as the MoCA, the Barthel Activities Daily Living Index, $\mathrm{mRS}$ and the National Institutes of Health Stroke Scale will be administered. Information about the locations of stroke lesions will also be collected.

\section{Data collection}

A research assistant will pay regular visits to the hospitals and community-based organisations to screen for potentially eligible participants. The research assistant will contact potential participants by phone and perform a preliminary eligibility assessment. The study aim, intervention, data collection procedure and rights to confidentiality will be explained to eligible patients. Participants' written informed consent will be obtained before commencing data collection. All consenting participants will be scheduled for baseline (T0) and follow-up assessment (T1, T2 and T3 immediately, 6 months and 12 months post-intervention, respectively).
Focus group interviews with the participants in the intervention group (at T1 and T3) and the facilitator (at T1) will be conducted. All interviews will be audiotaped.

\section{Analyses}

Data will be summarised and presented using the appropriate descriptive statistics. The normality of the continuous variables will be assessed using skewness and kurtosis statistics and normal probability plot. The appropriate transformations will be made on the skewed variables before subjecting them to inferential analysis if needed. The homogeneity of the participants' characteristics between the two study groups will be evaluated by independent $t$, chi-square or Fisher's exact tests, as appropriate. The outcome analysis will be performed in accordance with the intention-to-treat principle. The generalised estimating equations models will be used to compare the differential changes in each of the primary and secondary outcomes across time between the two groups. All of the statistical analyses will be performed using SAS release 9.4 (SAS Institute, Cary, NC). All statistical tests involved will be two-sided, with the level of significance set at 0.05 . All interview data will be transcribed verbatim. The transcripts will be coded and analysed by two independent researchers. The codes will be grouped to form major themes and sub-themes that correspond to the study aim and objectives.

\section{Ethical considerations}

The study has been approved by the Joint Chinese University of Hong Kong-New Territories East Cluster Clinical Research Ethics Committee (Ref. 2019.010). The research team will protect the participants' rights and safety by adhering to local laws, the Declaration of Helsinki, institutional policies, and the International Conference on Harmonisation-Good Clinical Practice. All eligible participants will be required to provide written consent.

\section{Discussion}

The NSI will fill the service gap by addressing young stroke survivors' ongoing psychological and skill-based needs to reintegrate into their social roles. Founding a complex intervention such as NSI on theoretical frameworks is crucial to explain the causal relationships between the intervention and the changes in outcomes. The results will enrich our knowledge of the practical application of integrating Narrative Theory and the principles of Self-efficacy and Outcome Expectation in the community reintegration of young stroke survivors. This novel RCT will test the short and long-term effects of a theory-based NSI on young stroke survivors' community reintegration and establish a new model of community 
reintegration after stroke to inform future research. The results will provide valuable evidence to develop clinical guidelines for survivors' community reintegration, which are not currently widely available. The NSI is expected to be integrated into existing stroke care services to benefit young stroke survivors in the long run.

\section{Supplementary Information}

The online version contains supplementary material available at https://doi. org/10.1186/s12883-020-02015-5.

\section{Additional file 1}

\section{Abbreviations}

HRQoL: Health-related Quality of Life; MoCA: Montreal Cognitive Assessment; mRS: modified Rankin Scale; NSI: Narrative and Skills-building Intervention; RCT: Randomised Controlled Trial; RNIL-C: Chinese version of the Reintegration to Normal Living Index

\section{Acknowledgements}

The authors would like to thank all of the young adults with stroke who have contributed to the development of the proposed intervention.

\section{Role of the funder}

The funding body has no role in the design of the study and collection, analysis, and interpretation of data and in writing the manuscript.

\section{Authors' contributions}

SHSL and JPCC conceived the idea for the study. SHSL, JPCC and KCC contributed to the research design and obtained funding for the study. EWCS, JHMY and SHL were involved in participant recruitment. SHSL wrote the first draft of the manuscript. All authors read and approved the final manuscript.

\section{Funding}

This study is supported by the Early Career Scheme of the University Grants Committee, Hong Kong SAR.

\section{Availability of data and materials}

Not applicable.

\section{Ethics approval and consent to participate}

The study has been approved by the Joint Chinese University of Hong KongNew Territories East Cluster Clinical Research Ethics Committee (Ref. 2019.010). All participants will provide written informed consent.

\section{Consent for publication}

Not applicable.

\section{Competing interests}

The authors declare that they have no competing interests.

\section{Author details}

${ }^{1}$ The Nethersole School of Nursing, Faculty of Medicine, Chung Chi College, The Chinese University of Hong Kong, Shatin, N.T., Hong Kong SAR, China. ${ }^{2}$ New Territories East Cluster, Hospital Authority, Hong Kong SAR, China. ${ }^{3}$ Department of Medicine, Alice Ho Miu Ling Nethersole Hospital, Hospital Authority, Hong Kong SAR, China. ${ }^{4}$ Department of Medicine, North District Hospital, Hospital Authority, Hong Kong SAR, China.

Received: 10 November 2020 Accepted: 25 November 2020 Published online: 04 January 2021

\section{References}

1. Feigin VL, Norrving B, Mensah GA. Global burden of stroke. Circ Res. 2017; 120(3):439-48.

2. Cameron Jl, O'Connell C, Foley N, Salter K, Booth R, Boyle R, Cheung D, Cooper N, Corriveau H, Dowlatshahi D, Dulude A, Flaherty P, Glasser E,
Gubitz G, Hebert D, Holzmann J, Hurteau P, Lamy E, LeClaire S, McMillan T, Murray J, Scarfone D, Smith EE, Shum V, Taylor K, Taylor T, Yanchula C, Teasell R, Lindsay P, Heart and Stroke Foundation Canadian Stroke Best Practice Committees. Canadian stroke best practice recommendations: managing transitions of care following stroke, guidelines update 2016. Int J Stroke. 2016;11(7):807-22.

3. Dworzynski K, Ritchie G, Fenu E, MacDermott K, Playford ED. Rehabilitation after stroke: summary of NICE guidance. BMJ. 2013;346:f3615.

4. Wood-Dauphinee SL, Opzoomer MA, Williams Jl, Marchand B, Spitzer WO. Assessment of global function: the reintegration to normal living index. Arch Phys Med Rehabil. 1988;69(8):583-90.

5. Lawrence M. Young adults' experience of stroke: a qualitative review of the literature. Br J Nurs. 2010;19(4):241-8.

6. Kuluski K, Dow C, Locock L, Lyons RF, Lasserson D. Life interrupted and life regained? Coping with stroke at a young age. Int J Qual Stud Health Wellbeing. 2014;9(1):22252.

7. Martinsen R, Kirkevold M, Sveen U. Younger stroke survivors' experiences of family life in a long-term perspective: a narrative hermeneutic phenomenological study. Nurs Res Pract. 2012;1-11.

8. Leahy DM, Desmond D, Coughlan T, O'Neill D, Collins DR. Stroke in young women: an interpretative phenomenological analysis. J Health Psychol. 2016;21(5):669-78.

9. Hinckley JJ. Telling the story of stroke when it's hard to talk. Top Lang Disord. 2015;35(3):258

10. Kirkevold M, Martinsen R, Bronken BA, Kvigne K. Promoting psychosocial wellbeing following stroke using narratives and guided self-determination: a feasibility study. BMC Psychol. 2014;2(1):4.

11. Corsten S, Schimpf EJ, Konradi J, Keilmann A, Hardering F. The participants' perspective: how biographic-narrative intervention influences identity negotiation and quality of life in aphasia. Int J Lang Commun Disord. 2015; 50(6):788-800

12. Pearce G, Pinnock H, Epiphaniou E, Parke HL, Heavey E, Griffiths CJ, Greenhalgh T, Sheikh A, Taylor SJC. Experiences of self-management support following a stroke: a meta-review of qualitative systematic reviews. PLoS One. 2015;10(12):e0141803.

13. Wolf TJ, Baum CM, Lee D, Hammel J. The development of the improving participation after stroke self-management program (IPASS): an exploratory randomized clinical study. Top Stroke Rehabil. 2016;23(4):284-92.

14. Lee D, Fischer H, Zera S, Robertson R, Hammel J. Examining a participationfocused stroke self-management intervention in a day rehabilitation setting: a quasi-experimental pilot study. Top Stroke Rehabil. 2017;24(8):601-7.

15. Stark S, Keglovits M, Somerville E, Hu Y, Conte J, Yan Y. Feasibility of a novel intervention to improve participation after stroke. Br J Occup Ther. 2018, 81(2):116-24.

16. Jones F, Riazi A, Norris M. Self-management after stroke: time for some more questions? Disabil Rehabil. 2013;35(3):257-64.

17. Parke HL, Epiphaniou E, Pearce G, Taylor SJ, Sheikh A, Griffiths CJ, Greenhalgh T, Pinnock H. Self-management support interventions for stroke survivors: a systematic meta-review. PLoS One. 2015;10(7):e0131448.

18. Wichowicz HM, Puchalska L, Rybak-Korneluk AM, Gasecki D, Wiśniewska A. Application of solution-focused brief therapy (SFBT) in individuals after stroke. Brain Inj. 2017;31(11):1507-12.

19. Satink T, Josephsson S, Zajec J, Cup EHC, de Swart BJM, Nijhuis-van der Sanden MWG. Self-management develops through doing of everyday activities-a longitudinal qualitative study of stroke survivors during two years post-stroke. BMC Neurol. 2016;16(1):221.

20. Satink T, Josephsson S, Zajec J, Cup EHC, de Swart BJM, Nijhuis-van der Sanden MWG. Negotiating role management through everyday activities: narratives in action of two stroke survivors and their spouses. Disabil Rehabil. 2016;38(24):2354-64.

21. Craig P, Dieppe P, Macintyre S, Michie S, Nazareth I, Petticrew M. Developing and evaluating complex interventions: the new Medical Research Council guidance. Int J Nurs Stud. 2013;50(5):587-92.

22. Sacco R, Kasner S, Broderick J, Caplan L, Connors JJ, Culebras A, Elkind MSV, George MG, Hamdan AD, Higashida RT, Hoh BL, Janis LS, Kase CS, Kleindorfer DO, Lee JM, Moseley ME, Peterson ED, Turan TN, Valderrama AL, Vinters HV. American Heart Association Stroke Council, Council on Cardiovascular Surgery and Anesthesia; Council on Cardiovascular Radiology and Intervention; Council on Cardiovascular and Stroke Nursing; Council on Epidemiology and Prevention; Council on Peripheral Vascular Disease; Council on Nutrition, Physical Activity and Metabolism: An updated 
definition of stroke for the 21st century: a statement for healthcare professionals from the American Heart Association/American Stroke Association. Stroke. 2013;44(7):2064-89.

23. Lo SHS, Chang AM, Chau JPC. Stroke self-management support improves survivors' self-efficacy and outcome expectation of self-management behaviors. Stroke. 2018;49(3):758-60.

24. Lee H, Fawcett J, DeMarco R. Storytelling/narrative theory to address health communication with minority populations. Appl Nurs Res. 2016;30:58-60.

25. Bandura A. Self-efficacy: toward a unifying theory of behavioral change. Psychol Rev. 1977;84(2):191-215.

26. Wood-Dauphinee SL, Opzoomer MA, Williams Jl, Marchand B, Spitzer WO Assessment of global function: the reintegration to Normal living index. Arch Phys Med Rehabil. 1988;69(8):583-90

27. Pang MY, Lau RW, Yeung PK, Liao L, Chung RC. Development and validation of the Chinese version of the reintegration to normal living index for use with stroke patients. J Rehabil Med. 2011;43(3):243-50.

28. Yesavage JA. The use of self-rating depression scales in the elderly. In: Poon LW, Crook T, Davis KL, Eisdorfer C, Gurland BJ, Kaszniak AW, Thompson LW, editors. Handbook for clinical memory assessment of older adults. Washington, DC: American Psychological Association; 1986. p. 213-7.

29. Chau J, Martin CR, Thompson DR, Chang AM, Woo J. Factor structure of the Chinese version of the geriatric depression scale. Psychol Health Med. 2006; 11(1):48-59.

30. Guerin JM, Copersino ML, Schretlen DJ. Clinical utility of the 15-item geriatric depression scale (GDS-15) for use with young and middle-aged adults. J Affect Disord. 2018;241:59-62.

31. Rule BG, Harvey HZ, Dobbs AR. Reliability of the geriatric depression scale for younger adults. Clin Gerontol. 2008;9(2):37-43.

32. Williams LS, Weinberger M, Harris LE, Clark DO, Biller J. Development of a stroke-specific quality of life scale. Stroke. 1999;30(7):1362-9.

33. Lo SHS, Chang AM, Chau JPC. Establishing equivalence of a Chinese version of the stroke specific quality of life measure for stroke survivors. Disabil Rehabil. 2017:39(11):1079-86.

34. Jones F, Partridge C, Reid F. The stroke self-efficacy questionnaire: measuring individual confidence in functional performance after stroke. J Clin Nurs. 2008;17(7B):244-52.

35. Lo SHS, Chang AM, Chau JPC. Translation and validation of a Chinese version of the stroke self-efficacy questionnaire in community-dwelling stroke survivors. Top Stroke Rehabil. 2016;23(3):163-9.

\section{Publisher's Note}

Springer Nature remains neutral with regard to jurisdictional claims in published maps and institutional affiliations.

Ready to submit your research? Choose BMC and benefit from:

- fast, convenient online submission

- thorough peer review by experienced researchers in your field

- rapid publication on acceptance

- support for research data, including large and complex data types

- gold Open Access which fosters wider collaboration and increased citations

- maximum visibility for your research: over $100 \mathrm{M}$ website views per year

At $\mathrm{BMC}$, research is always in progress.

Learn more biomedcentral.com/submissions 